Pacific Journal of Mathematic 


\title{
ON A CLASS OF DIFFERENTIAL EQUATIONS FOR VECTOR-VALUED DISTRIBUTIONS
}

\author{
H. O. FATTORINI
}

The aim of this paper is to seek necessary and sufficient conditions on the linear operator $A$ in a linear topological space in order that the Cauchy problem for the equation $U^{(\alpha)}-A U=T$ should be well set in the sense of distributions (see definition in $\S 2$ ). Here $0<\alpha<\infty, U^{(\alpha)}$ is the fractional derivative of $U$ of order $\alpha$. Such conditions are obtained for $\alpha$ integer $\geqq 3$ and then for any $\alpha>0$, this time with the additional assumption of (at most) exponential growth of the solutions at infinity.

Throughout this paper $E$ will be a quasi-complete, barreled locally convex linear topological space over the field $C$ of complex numbers ([1], Chapter II, §4; [2], Chapter II, §1 and §2), $A$ a closed linear operator with domain $D(A)$ dense in $E$ and range in $E$.

The equation

$$
u^{(\alpha)}(t)=A u(t)
$$

was studied in [3]. If $\alpha$ is an integer $\geqq 3$ and the Cauchy problem for (1.1) is "well posed" (strong solutions exist for a dense set of initial data, are unique and depend continuously on them) it was shown that

(a) $D(A)=E$ and $A$ is continuous.

(b) The series $\sum_{j=0}^{\infty} t^{j} A^{j} u /(\alpha j)$ ! converges in $E$ for all $t, u$. The solutions of (1.1) are actually holomorphic and can be expressed as $u(t)=\sum_{k=0}^{\alpha-1} S_{k}(t) u^{(k)}(0)$, where $S_{k}(t)=\sum_{j=0}^{\infty} t^{\alpha j+k} A^{j} u /(\alpha j+k) !, 0 \leqq$ $k \leqq \alpha-1$. Conversely, conditions (a) and (b) imply that the Cauchy problem for (1.1) is well posed (see [3], Th. 3.1). A necessary and sufficient condition for the solutions of (1.1) to increase at most exponentially at $\infty$ is the existence of $R(\lambda ; A)$ for large $|\lambda|$ and its analyticity at $\infty$ ([3], 3.3). It has been suggested by J. L. Lions that the above results will still hold if we only assume the Cauchy problem for (1.1)-or, rather, for its inhomogeneous version-to be well set in the sense of distributions (this notion was introduced by him in [7] for the case $\alpha=1$ ). Lions also raised the question of whether the results could be extended to the case of noninteger $\alpha>2$. We give here some partial answers to these questions. Under a special assumption, Theorem 3.1 of [3] is extended to the distribution setting, although only for $\alpha$ integer; Theorem 3.3 is also extended for all values of $\alpha>2$. (Theorems 4.1 and 5.2 respectively.) We also examine the case 
$\alpha<2$, always with the assumption of exponential increase at infinity and give conditions on $R(\lambda ; A)$ that insure the Cauchy problem to be well set (Theorem 6.1). For $\alpha=1$, we obtain the condition of Lions for generation of distribution semigroups.

2. The Cauchy problem. We denote by $R$ the real numbers. The symbol $\mathscr{E}$ stands for a family $\{|\cdot|, \cdots\}$ of semi-norms determining the topology of $E$ (see [1], Chapter II, $\S 4$; for instance, $\mathscr{E}$ can be taken as the family of all continuous semi-norms in $E$ ), i.e., such that the (generalized) sequence $\left\{u_{r}\right\}$ converges to zero if and only if $\left|u_{\gamma}\right| \rightarrow 0$ for all $|\cdot| \in \mathscr{E}$. We assume $D(A)$, the domain of $A$ endowed with the topology generated by the semi-norms $u \rightarrow|u|, u \rightarrow|A u|$, $|\cdot| \in \mathscr{E} . \quad D(A)$ is under this topology a quasi-complete locally convex linear topological space.

In the following remarks the spaces $F, G, \cdots$ are as $E$, quasicomplete, barreled locally convex linear topological spaces. The space $\mathscr{L}(F, G)$ consists of all linear continuous operators from $F$ into $G$ endowed with the topology of uniform convergence on bounded sets of $F . \mathscr{L}(F, G)$ is a locally convex, quasi-complete linear topological space (see [2], Chapter III, $\S 3$, no. 7 ; it is not necessary for this result that $G$ be barreled). We shall write $\mathscr{L}(F)$ instead of $\mathscr{L}(F, F)$, $F^{*}$ instead of $\mathscr{L}(F, C)$, application of an element $u^{*} \in F^{*}$ to $u \in F$ being denoted by $\left\langle u^{*}, u\right\rangle$ or $\left\langle u, u^{*}\right\rangle$.

We recall that the "equi-continuity principle" ([2], Chapter III, $\S 3$, Théorème 2) holds in $\mathscr{L}(F, G)$ (thus in particular in $\left.\mathscr{L}(F), F^{*}\right)$; if $\left\{B_{r}\right\}$ is a family of elements of $\mathscr{L}(F, G)$ such that $\left\{B_{r} u\right\}$ is bounded in $G$ that is, such that $\sup _{r}\left|B_{r} u\right|<\infty$ for any continuous seminorm $|\cdot|$ in $G$ for every $u \in F$, then $\left\{B_{r}\right\}$ is an equicontinuous family. This principle will be used many times in what follows, sometimes without explicit mention.

The space $\mathscr{D}_{t}$ (or simply $\mathscr{D}$ ) consists of all complex-valued functions $t \rightarrow \varphi(t)$ defined in $R$, infinitely differentiable there and with compact support; the space $\mathscr{D}_{s, t}$ is similarly defined but with reference to functions $(s, t) \rightarrow \varphi(s, t)$ of two variables. Both spaces will be endowed with their L. Schwartz topologies ([10], Chapter III). $\mathscr{D}_{0}$ consists of all $\varphi \in \mathscr{D}$ with support in $(0, \infty)$. By definition, a generalized sequence $\left\{\varphi_{\gamma}\right\}$ in $\mathscr{D}_{0}$ converges to zero if and only if the supports of the $\varphi_{\gamma}$ are contained in a fixed compact subset of $(0, \infty)$ and $\varphi_{r}^{(m)}(t) \rightarrow 0$ uniformly in $(0, \infty)$ for all $m \geqq 0$. The space $\mathscr{D}^{\prime}(F)$ (or $\left.\mathscr{D}_{t}^{\prime}(F)\right)$ of $F$-valued distributions of one variable is $\mathscr{L}(\mathscr{D} ; F)$; similarly, $\mathscr{D}_{s, t}^{\prime}(F)=\mathscr{L}\left(\mathscr{D}_{s, t} ; F\right)$. The space $\mathscr{D}_{0}^{\prime}(F)(F$-valued distributions defined in $(0, \infty))$ is $\mathscr{L}\left(\mathscr{D}_{0}, F\right)$. For any real $a$, the subspace of $\mathscr{D}^{\prime}(F)$ consisting of distributions with support in $[a, \infty)$ will be denoted 
by $\mathscr{D}_{[a, \infty)}^{\prime}(F)$; it inherits the topology of $\mathscr{D}^{\prime}(F)$. Finally,

$$
\overline{\mathscr{D}}_{+}^{\prime}(F)=\bigcup_{n=-\infty}^{\infty} \mathscr{D}_{[n, \infty)}^{\prime}(F)^{1}
$$

and we assign to $\overline{\mathscr{D}}_{+}^{\prime}(F)$ the inductive limit of the topologies of the $\mathscr{D}_{[n, \infty)}^{\prime}(F)$ ([1], Chapter II, §4). As customary, we write $\mathscr{D}^{\prime}(C)=$ $\mathscr{D}^{\prime}$ and similarly for other distribution spaces.

Let $S$ be a distribution in, say, $\overline{\mathscr{D}}_{+}^{\prime}(\mathscr{L}(E, F)), T \in \overline{\mathscr{D}}_{+}^{\prime}(E)$. We recall briefly the definition of the convolution $S * T$ as given in [9] (Proposition 39, p. 167). It is a consequence of Proposition 33, p. 145, that there exists a unique distribution $V \in \mathscr{D}_{s, t}^{\prime}(F)$ such that

$$
V(\varphi \otimes \psi)=S(\varphi) T(\psi)
$$

for any $\varphi, \psi \in \mathscr{D}$ (here $\varphi \otimes \psi$ denotes the function in $\mathscr{D}_{s, t}$ defined by $(\varphi \otimes \psi)(s, t)=\varphi(s) \psi(t))$ and whose support is contained in the Cartesian product $\operatorname{supp}(S) \times \operatorname{supp}(T) . \quad S * T$ is then defined by

$$
(S * T)(\varphi)=V(\widehat{\varphi})
$$

for any $\varphi \in \mathscr{D}$, where $\hat{\varphi}(s, t)=\varphi(s+t)$ (note that, since the intersection of the supports of $V$ and $\hat{\varphi}$ is compact the expression $V(\hat{\varphi})$ has a sense although $\hat{\varphi} \notin \mathscr{D}_{s, t}$ if $\varphi \neq 0$ ). For any $S, T$ we have

$$
\operatorname{supp}(S * T) \subseteq \operatorname{supp}(S)+\operatorname{supp}(T) ;
$$

the convolution, as a linear map (we assume $S$ fixed) from $\overline{\mathscr{D}}_{+}^{\prime}(\mathscr{L}(E, F)) \times \overline{\mathscr{D}}_{+}^{\prime}(E)$ into $\overline{\mathscr{D}}_{+}^{\prime}(E)$ is continuous (see again [9], Proposition 39 for proofs of these and other facts). The convolution $S * T$ can be defined in a similar way when $S \in \overline{\mathscr{D}}_{+}^{\prime}$ and $T \in \overline{\mathscr{D}}_{+}^{\prime}(F)$, when $S \in \mathscr{\mathscr { D }}_{+}^{\prime}(\mathscr{L}(E, F))$ and $T \in \overline{\mathscr{D}}_{+}^{\prime}(\mathscr{L}(G, E)), \cdots$ and enjoys the same properties as in the previous case.

Fractional derivatives will be defined by means of convolutions. For any complex $\beta$ we write

$$
Y_{\beta}=\frac{1}{\Gamma(\beta)} \mathrm{Pf} .\left(t^{\beta-1}\right)_{t>0} \cdot
$$

This distribution (see [10], Chapter II, § II, p. 43) coincides with the function $(h(t) t)^{\beta-1} / \Gamma(\beta)$ for $\operatorname{Re} \beta>0, h$ the Heaviside function $(h(t)=0$ for $t<0, h(t)=1$ for $t>1$ ). The function of $\beta$ that results applying it to any $\varphi \in \mathscr{D}$ admits of an analytic extension to the entire

${ }^{1}$ Let $\mathscr{D}$ - be the space of all infinitely differentiable functions in $R$ with support bounded above endowed with its usual Schwartz topology ([10], Chapter VI, \&5, p. 172). Then $\overline{\mathscr{D}}_{+}^{\prime}(F) \subseteq \mathscr{D}{ }_{+}(F)=\mathscr{f}\left(\mathscr{D}_{-}, F\right)$ algebraic and topologically. The reverse inclusion is true if, say, $F$ is a Banach space but not in general. See [8], p. 62, where a similar situation is discussed with reference to distributions with compact support. 
plane, and its value at a given $\beta$ is taken as definition of $Y_{\beta}(\phi)$ (see [10], loc. cit. for details). For any $\beta, Y_{\beta} \in \mathscr{D}^{\prime}$ and its support is contained in $[0, \infty)$. If $U \in \overline{\mathscr{D}}_{+}^{\prime}(F), 0 \leqq \alpha<\infty$ we define

$$
U^{(\alpha)}=\text { derivative of order } \alpha \text { of } U=Y_{-\alpha} * U
$$

([10], Chapter VI, §5, p. 174); the definition is justified by the fact that $Y_{-n}=\delta^{(n)}, n=0,1, \cdots$. If $U=0$ for $t<a$, the same it true of $U^{(\alpha)}$.

Finally, some notational conventions. If, say, $S \in \mathscr{D}^{\prime}(\mathscr{L}(E, F))$, $u \in E$, we denote by $S u$ the distribution (in $\mathscr{D}^{\prime}(F)$ ) defined by $(S u)(\varphi)=$ $S(\varphi) u, \varphi \in \mathscr{D}$. Similar definition for $A S$, where $A \in \mathscr{L}(F, G)$. Following [8], p. 51, if $T \in \mathscr{D}^{\prime}, u \in F, T \otimes u$ is the distribution in $\mathscr{D}^{\prime}(F)$ given by $(T \otimes u)(\varphi)=T(\varphi) u$. We shall use the same notation for an $F$-valued function and for the distribution (in $\mathscr{D}^{\prime}(F)$ ) that it defines.

Definition 2.1. Let $0<\alpha<\infty$. The Cauchy problem for the equation

$$
U^{(\alpha)}-A U=T
$$

is well set (in the sense of distributions) if and only if

(a) (Existence) For every $T \in \overline{\mathscr{D}}_{+}^{\prime}(E)$ there exists a solution $U \in \overline{\mathscr{D}}_{+}^{\prime}(D(A))$ of (2.1).

(b) (Uniqueness) Let $U \in \overline{\mathscr{D}}_{+}^{\prime}(D(A))$ be a solution of (2.1) with $T \in \overline{\mathscr{D}}_{+}^{\prime}(E)$. Assume $T=0$ if $t<a$. Then $U=0$ for $t<a$.

(c) (Continuous dependence) Let $\left\{T_{\gamma}\right\}$ be a generalized sequence of elements of $\overline{\mathscr{D}}_{+}^{\prime}(E)$ with $T_{\gamma} \rightarrow 0$ in $\mathscr{D}^{\prime}(E), T_{\gamma}=0$ for $t<a(a>-\infty)$ for all $\gamma$. Let $U_{\gamma} \in \overline{\mathscr{D}}_{+}^{\prime}(D(A))$ be the corresponding solutions of (2.1). Then $U_{r} \rightarrow 0$ in $\mathscr{D}^{\prime}(D(A))$.

A few comments on (b) and (c) will be useful later. Observe first that (b) implies

(b') Let $u(\cdot)$ be an infinitely differentiable $D(A)$-valued function vanishing for large negative $t$ and such that

$$
u^{(\alpha)}(t)-A u(t)=0
$$

for $t \leqq 0$. Then $u(t)=0$ for $t \leqq 0$.

It is also true that $\left(b^{\prime}\right)$ implies (b). To see this, let $U, T$ be the two distributions of $(b), \varphi \in \mathscr{D}$ with support in $(-\infty, a)$. Define

$$
\varphi_{t}(s)=\varphi(s-t),(I \varphi)(s)=\varphi(-s) \text {. }
$$

Then if $u(t)=U\left(\varphi_{t}\right)=(U * I \phi)(t), u(\cdot)$ is a $C^{\infty}, D(A)$-valued function and $u^{(\alpha)}(t)-A u(t)=T\left(\varphi_{t}\right)$. Since $T\left(\varphi_{t}\right)=0$ for $t<0$ ( $T$ is zero for $t<a)$, we have $u(0)=U(\varphi)=0$, which shows that $U$ itself is zero 
for $t<a$ as claimed.

By definition of the inductive limit topology ([1], Chapter II, $\S 4$, $\left.n^{\circ} 6\right)$ a generalized sequence $\left\{T_{\gamma}\right\}$ in $\overline{\mathscr{D}}_{+}^{\prime}(F)$ converges to zero if and only if $T_{r} \rightarrow 0$ in $\mathscr{D}^{\prime}(F)$ and all the $T_{r}^{\prime}$ s are contained in a fixed $\mathscr{D}_{[a, \infty)}^{\prime}(F)$ (that is, if $T_{\gamma}=0$ for all $\gamma$ and $t<$ some fixed $a$ ). This shows that (c) amounts to the assertion that the map

$$
T \rightarrow U
$$

from $\overline{\mathscr{D}}_{+}^{\prime}(E)$ to $\overline{\mathscr{D}}_{+}^{\prime}(D(A))$ given by the equation (2.1) (which map, by virtue of (a) and (b) is well-defined and linear) is continuous. It is also plain that the map (2.2) commutes with translations. We deduce more information about (2.2) by means of the following result.

AUXILIARY Lemma 2.2 Let $\mathscr{C l}$ be a linear continuous operator from $\overline{\mathscr{D}}_{+}^{\prime}(F)$ to $\overline{\mathscr{D}}_{+}^{\prime}(G)$ commuting with translations. Assume, moreover that $\mathscr{C l} T=0$ in $t<a$ whenever $T=0$ in $t<a$. Then there exists $S \in \overline{\mathscr{D}}_{+}^{\prime}(\mathscr{L}(F, G))$ with support contained in $t \geqq 0$ such that

$$
\mathscr{C l} T=S * T \text {. }
$$

The proof is identical to that of the "scalar-valued" theorem ([10], Chapter VI, §3, p. 162; see also [7], p. 150 for the Banach space case). We define a distribution $S \in \mathscr{D}^{\prime}(\mathscr{L}(F, G))$ by the formula $S(\varphi) u=(\mathscr{l}(\delta \otimes u))(\varphi), \varphi \in \mathscr{D}, u \in F ;$ since $\operatorname{supp} \mathscr{l}(\delta \otimes u) \leqq[0, \infty)$, $\operatorname{supp}(S) \subseteq[0, \infty)$. Then $\mathscr{N} U=S * U$ is a linear continuous operator from $\overline{\mathscr{D}}_{+}^{\prime}(F)$ into $\overline{\mathscr{D}}_{+}^{\prime}(G)$. (See the previous remarks on convolution.) We only need now to verify the equality $\mathscr{C}=\mathscr{N}$ for distributions $U$ of the form $\tau_{a} \delta \otimes u, \tau_{a}$ the operator of translation by $a, u \in F^{(2)}$. But $\mathscr{C}\left(\tau_{a} \delta \otimes u\right)=\tau_{a} \mathscr{C}(\delta \otimes u)=\tau_{a}(S u)=S *\left(\tau_{a} \delta \otimes u\right)=\mathscr{N}\left(\tau_{a} \delta \otimes u\right)$, thus our result is proved.

Return now to the map $/ C$ given by (2.2). The distribution $S \in \overline{\mathscr{D}}_{+}^{\prime}(\mathscr{L}(E, D(A))$ corresponding to $\mathscr{C l}$ will be called the propagator of 2.1. It follows from its definition that it satisfies the equation

$$
S^{(\alpha)}-A S=\delta \otimes I \text {. }
$$

We prove now a few simple properties of $S$.

Lemma 2.3. The operators $S(\varphi), S(\psi), A$ commute for any

${ }^{2}$ The subspace of $\mathscr{D}^{\prime}(F)$ generated by all elements of the form $U \otimes u, U \in \mathscr{D}^{\prime}$ $u \in F$ can be identified with the tensor product $\mathscr{D}^{\prime} \otimes F\left([8]\right.$, p. 50). But $\mathscr{D}^{\prime} \otimes F$ is dense if $\mathscr{D}^{\prime}(F)$; on the other hand, the subspace of $\mathscr{D}^{\prime}$ generated by all elements of the form $\tau_{a} \delta, a \in R$ is dense in $\mathscr{D}^{\prime}$ ([10], Chapter II, \&2, p. 75) so that the subspace of $\mathscr{D}^{\prime}(F)$ generated by all elements of the form $\tau_{a} \delta \otimes u, a \in R, u \in F$ is dense in $\mathscr{D}^{\prime}(F)$. 
$\varphi, \psi \in \mathscr{D}$.

Proof. Let $u \in D(A)$. Since $U=A(S u)=(S u)^{(\alpha)}-\delta \otimes u$, it is clear that $U \in \overline{\mathscr{D}}_{+}^{\prime}(D(A))$; moreover

$$
U^{(\alpha)}-A U=A\left((S u)^{(\alpha)}\right)-A(A(S u))=A(\delta \otimes u)=\delta \otimes A u .
$$

By uniqueness, $U=S(A u)$, i.e.,

$$
A(S u)=S(A u)
$$

which shows that $S(\varphi)$ and $A$ commute for any $\varphi \in \mathscr{D}$. As for commutativity of $S(\varphi), S(\psi)$ one only needs to observe that

$$
V=(S(\psi) S) *(\delta \otimes u)=S(\psi)(S u)
$$

is a solution of $V^{(\alpha)}-A V=\delta \otimes S(\psi) u$ and reason as before.

Lemma 2.4. Let $\alpha=n$ be an integer $\geqq 1, \varphi, \psi \in \mathscr{D}_{0}$. Then

$$
S(\varphi * \psi)=\sum_{k=0}^{n-1} S^{(k)}(\varphi) S^{(n-1-k)}(\psi) .
$$

The proof is a modification of one of Lions ([7], Théorème 5.1, p. 149) for the case $n=1$. Let $(I \varphi)(t)=\varphi(-t)$ for any $\varphi \in \mathscr{D}$. Take now $\varphi, \psi \in \mathscr{D}_{0}$. Let $U, W, V_{1}, \cdots, V_{n}$ be the solutions in $\overline{\mathscr{D}}_{+}^{\prime}(D(A))$ of the equations

$$
\begin{gathered}
U^{(n)}-A U=I \psi \otimes u \\
W^{(n)}-A W=(I \phi * I \psi) \otimes u \\
V_{k}^{(n)}-A V_{k}=(I \varphi)^{(k)} \otimes U^{(n-1-k)}(0) \quad(0 \leqq k \leqq n-1),
\end{gathered}
$$

$u \in E$. (Observe that $U, W, V_{1}, \cdots, V_{n}$ are obtained by convolution of the propagator with the right-hand members of (2.5), (2.6), (2.7) thus they are all $C^{\infty}$ functions.)

Let now $h$ be the Heaviside function. A simple computation shows that

$$
(h U)^{(n)}=\sum_{k=0}^{n-1} \delta^{(n-1-k)} \otimes U^{(k)}(0)+h U^{(n)} .
$$

Taking now into account the fact that $U$ satisfies (2.5) and that $\operatorname{supp}(I \psi) \subset(-\infty, 0), \quad(h U)^{(n)}-A(h U)=\sum_{k=0}^{n-1} \delta^{(n-1-k)} \otimes U^{(k)}(0)$. Then by virtue of (2.7) we get, by uniqueness

$$
I \varphi * h U=\sum_{k=0}^{n-1} V_{k} .
$$


Similarly,

$$
I \varphi * U=W .
$$

Observe, finally, that since $\operatorname{supp}(I \varphi) \subset(-\infty, 0)$

$$
(I \phi * h U)(0)=(I \phi * U)(0) \text {. }
$$

Consequently

$$
\begin{aligned}
S(\varphi * \psi) u & =(S *(I(\varphi * \psi))(0) u=(S *(I \varphi * I \psi))(0) u=W(0) \\
& =(I \varphi * U)(0)=(I \varphi *(h U))(0)=\sum_{k=0}^{n-1} V_{k}(0) \\
& =\sum_{k=0}^{n-1}\left(S *\left((I \varphi)^{(k)}\right)\right)(0) U^{(n-1-k)}(0) \\
& =\sum_{k=0}^{n-1} S^{(k)}(\varphi)\left(S^{(n-1-k)} *(I \psi \otimes u)\right)(0) \\
& =\sum_{k=0}^{n-1} S^{(k)}(\varphi) S^{(n-1-k)}(\psi) u \text { as claimed }
\end{aligned}
$$

3. Some regularity results. The results in this section say, roughly speaking, that if $u$ belongs to a set of "smooth elements" of $E$ then $S u$ will actually be a $C^{\infty}$ function in $t \geqq 0$; moreover, if $u_{\gamma} \rightarrow 0$ "rapidly enough," then $S u_{\gamma}$ will converge to zero in a topology considerably stronger than that of $\mathscr{D}^{\prime}(E)$. We also examine certain smooth solutions of (2.1). As in the last part of the previous section we assume $\alpha=n=$ integer $\geqq 1$.

We introduce at this point a special hypothesis on $S$, namely

Assumption 3.1. $S$ is a distribution of finite order locally, ${ }^{(3)}$ which will be assumed to hold throughout the rest of this section (as well as in $\S 4)$.

Recall ([8], Proposition 24, p. 86) that, under the preceding hypothesis, if $\Omega$ is any open bounded interval in $R$ then there exists a continuous $\mathscr{L}(E, D(A))$-valued function defined in $\Omega$ and such that

$$
S=f^{(p)} \text { in } \Omega
$$

(the integer $p \geqq 0$ may depend on $\Omega$ ).

Let $D$ be the subspace of $E$ consisting of all $u \in E$ such that $S u$

${ }^{3}$ Assumption 3.1 is unnecessary whenever $E$ is a Banach space-or, more generally, when $£(E D(A))$ is a $(D F)$-space $([\mathbf{8}], \S 3)$. It is also unnecessary, with no special restriction on $E$, when $n=1$; for the solution of $U^{\prime}-A U=\delta \otimes S(\varphi) u, \varphi \in \mathscr{D}_{0}, u \in E$ is $U=h V, V$ the $\left(C^{\infty}\right)$ solution of $V^{\prime}-A V=I \varphi \otimes u$ ([7], p. 152), which allows one to establish all the following regularity results. We do not know whether Assumption 3.1 can be altogether eliminated in all cases. 
coincides with a $D(A)$-valued function $g(t)$, infinitely differentiable in $t>0$ and such that $g^{(m)}(0+)=\lim _{t \rightarrow 0+} g^{(m)}(t)$ exists for all $m \geqq 0$ (all in the topology of $D(A)$ ). For $u \in D$ we define $G(t) u=g(t)$. For fixed $t \geqq 0 G(t)$ is a linear operator in $E$ with domain $D$.

Lemma 3.2. $D=D\left(A^{\infty}\right)=\bigcap_{m=1}^{\infty} D\left(A^{m}\right)$.

Proof. Let $u \in D\left(A^{\infty}\right)$. Consider the identity

$$
S^{(n)} u-A(S u)=\delta \otimes u
$$

(which is a simple consequence of the definition of $S$ ). Differentiating (3.2) repeatedly and making use at each step of the commutativity of $A$ and $S$ (Lemma 2.3) we obtain for $m \geqq 1$.

$$
\begin{aligned}
& S^{(m n)} u=A\left(S^{(m-1) n} u\right)+\delta^{(m-1) n} \otimes u=S^{(m-1) n} A u \\
& \quad+\delta^{(m-1) n} \otimes u=S^{(m-2) n} A^{2} u+\delta^{(m-1) n} \otimes u \\
& \quad+\delta^{(m-2) n} \otimes A u=\cdots=S A^{m} u+\sum_{k=}^{m-1} \delta^{(k n)} \otimes A^{m-1-k} u .
\end{aligned}
$$

Let now $\Omega$ be an open interval in $R$ (say $(-a, a), 0<a<\infty), f$ the function associated with $S$ in $\Omega$ by (3.1). We have

$$
S^{(m n)} u=f^{(p)} A^{m} u+\sum_{k=0}^{m-1} \delta^{(k n)} \otimes A^{m-1-k} u
$$

in $\Omega$. Choose a $m$ with $m n>p$ and integrate the differential equation (3.3). We obtain

$$
S^{(m n-p)} u=f A^{m} u+\sum_{k=0}^{m-1} Y_{p-k n} \otimes A^{m-1-k} u+P_{m, p}
$$

in $\Omega, Y_{\beta}$ the distribution $\in \mathscr{D}^{\prime}$ defined in $\S 2$, (here we are using the fact that $\left.Y_{0}=\delta, Y_{\alpha}^{(\beta)}=Y_{\alpha-\beta}\right), P_{m, p}$ a polynomial of degree $\leqq p-1$ with coefficients in $D(A)$. Since $m$ is arbitrary, it is clear that $S u$ coincides with a $C^{\infty}$ function in $\{t \in \Omega ; t>0\}$; reasoning in this way for any $\Omega$ we see that $u \in D$.

Conversely, assume $u \in D$, and let $g(t)=G(t) u$, for $t \geqq 0, U_{k}(t)=$ $g^{(k)}(t)$ for $t \geqq 0, U_{k}(t)=0$ for $t<0$. Call $U=U_{0}$. Then we have

$$
\begin{aligned}
U^{\prime} & =\delta \otimes U(0)+U_{1}, \cdots \\
U^{(n)} & =\sum_{k=1}^{n-1} \delta^{(n-1-k)} \otimes U^{(k)}(0)+U_{n} .
\end{aligned}
$$

Observe now that $U=S u$ satisfies $U^{(n)}-A U=\delta \otimes u$; since $U$ is $C^{\infty}$ in $t>0$ it satisfies $U^{(n)}(t)=A U(t)$ there. Consequently $\delta \otimes u=$ $U^{(n)}-A U=\sum_{k=0}^{n-1} \delta^{(n-1-k)} \otimes U^{(k)}(0)$; equating coefficients we obtain 


$$
g(0+)=\cdots=g^{(n-2)}(0+)=0, g^{(n-1)}(0+)=u .
$$

Observe next that for all $m \geqq 0$ we have

$$
g^{(m+n)}(t)=A g^{(m)}(t), t>0 .
$$

(This is obtained by differentiating the equality for $m=0$.) Taking $m=n-1$ and letting $t \rightarrow 0$ we obtain $A u=A g^{(n-1)}(0+)=g^{(2 n-1)}(0+)$ which belongs to $D(A)$; then $u \in D\left(A^{2}\right)$ and $A^{2} u=A g^{(2 n-1)}(0+)=$ $g^{(3 n-1)}(0+), \cdots$ etc. An examination of the initial values of $g$ readily shows

Corollary 3.3. Let $u \in D$. Then $G^{(k)}(0+) u=0$ if $k \neq m n-1$, $k \geqq 0 G^{(m n-1)}(0+) u=A^{m-1} u, m \geqq 1$.

Our next step is to show that $D=D\left(A^{\infty}\right)$ contains "enough" elements. Observe first that if $\varphi \in \mathscr{D}_{0}$ then $S(\varphi) u \in D\left(A^{\infty}\right)$ for any $u \in E$; for, since $S^{(n)} u-A(S u)=\delta \otimes u$ and $\operatorname{supp}(\varphi) \subset(0, \infty), A S(\varphi)=$ $S^{(n)}(\varphi) u$, thus $A S(\varphi) u \in D(A)$ and $A^{2} S(\varphi) u=S^{(2 n)}(\varphi) u$. Repeating the preceding reasoning we see that $S(\varphi) u \in D\left(A^{m}\right)$ for any $m \geqq 0$ and

$$
A^{m} S(\varphi) u=S^{(m n)}(\varphi) u \text {. }
$$

Lemma 3.4. $D$ is dense in $E$.

We shall actually show a stronger result, namely that the subspace generated by $D_{0}=\left\{v \in E ; v=S(\varphi) u, \phi \in \mathscr{D}_{0}, u \in E\right\}$ is dense in $D(A)$. Assume this is false. Then there exists $u^{*} \in D\left(A^{*}\right)$ such that $\left\langle u^{*}, S(\varphi) u\right\rangle=\left\langle S(\varphi)^{*} u^{*}, u\right\rangle=0$ for all $u \in E, \varphi \in \mathscr{D}_{0}$, i.e.,

$$
S(\varphi)^{*} u^{*}=0
$$

for all $\varphi \in \mathscr{D}_{0}$, where $S(\varphi)^{*}: D(A)^{*} \rightarrow E^{*}$ denotes the operator adjoint to $S(\varphi)$. Let now $K$ be any bounded set in $E$ and let $\phi \in \mathscr{D}$. Since

$$
\sup _{u \in K}\left|\left\langle S(\varphi)^{*} u^{*}, u\right\rangle\right|=\sup _{u \in K}\left|\left\langle u^{*}, S(\varphi) u\right\rangle\right|
$$

it follows from the fact that $S$ is a $\mathscr{L}(E, D(A))$-valued distribution and from the definition of the topology of $E^{*}$ that $\varphi \rightarrow S(\varphi)^{*} u^{*}=$ $U(\varphi)$ belongs to $\mathscr{D}^{\prime}\left(E^{*}\right)$. By applying $u^{*}$ to elements of $E$ of the form $(-1)^{n} S\left(\varphi^{(n)}\right) u-S(\varphi) A u=(-1)^{n} S\left(\phi^{(n)}\right) u-A S(\varphi) u=\varphi(0) u, u \in D(A)$, we also see that $U$ satisfies the equation

$$
U^{(n)}-A^{*} U=\delta \otimes u^{*}
$$

where $A^{*}: E^{*} \rightarrow D(A)^{*}$ is the adjoint of $A .^{4}$ Since $S$ has its support

${ }^{4}$ Since $D(A) \cong E$ algebraically and topologically and $D(A)$ is dense in $E$, we can identify $E^{*}$ with a subspace of $D(A)^{*}$, and the inclusion $E^{*} \subseteq D(A)^{*}$ is also topologic. 
in $t \geqq 0$, so does $U$; but, since the vanishing of the expression (3.6) for all $\varphi \in \mathscr{D}_{0}$ means that $U$ is zero for $t>0$ we see that $\operatorname{supp}(U)$ reduces to the point 0 .

Let now $\Omega$ be an interval around the origin, $f$ a $\mathscr{L}(E, D(A))$ valued function satisfying (3.1) in $\Omega$ for some $p \geqq 0$. The function $g=f^{*} u^{*}$ takes values in $E^{*}$, is continuous in $\Omega$ and satisfies

$$
U=g^{(p)}
$$

in $\Omega$. Since $U$ is zero both for $t<0$ and $t>0, g(t)=P(t)$ for $t>0$, $g(t)=Q(t)$ for $t<0$, both $P$ and $Q$ being polynomials of degree $\leqq p-1$ with coefficients in $E^{*}$ and such that $P(0)=Q(0)$.

Consider now the different values of $p$. If $p=0, g=U=0$ and there is nothing to prove. If $p=1, g$ is constant in $\Omega$ and again $U=0$. Finally, if $p \geqq 2 U$ has to be of form

$$
U=\sum_{k=0}^{m} \delta^{(k)} \otimes u_{k}^{*}
$$

where $m=p-2, u_{0}^{*}, \cdots, u_{m}^{*}$ elements of $E^{*} .^{5}$ Replacing now this expression for $U$ in the equation (3.7) we get

$$
\sum_{k=0}^{m} \delta^{(n+k)} \otimes u_{k}^{*}=\sum_{k=0}^{m} \delta^{(k)} \otimes A^{*} u_{k}^{*}+\delta \otimes u^{*} .
$$

Let now $q \geqq 0$ such that $q n \leqq m<(q+1) n$. By equating coefficients in (3.10) we easily obtain that

$$
u^{*}=-A u_{0}^{*}, u_{0}^{*}=A^{*} u_{n}^{*}, \cdots, u_{(4-1) n}^{*}=A^{*} u_{q n}^{*}, u_{q n}^{*}=0
$$

which shows $u^{*}=0$.

Lemma 3.5. Let $\left\{\varphi_{r}\right\}$ be a generalized sequence in $\mathscr{D}_{0}$ convergent to some element $\varphi_{0} \in \mathscr{D}_{0}$ in the topology of $\mathscr{D}, v$ any element of $E$. Then $G(\cdot) S\left(\varphi_{i}\right) v$ converges uniformly to $G(\cdot) S\left(\varphi_{0}\right) v$ on compacts of $t \geqq 0$ together with all its derivatives.

Proof. Assume-as we may-that $\varphi_{0}=0$. Let $\Omega$ be an open set containing the origin and let $f$ be the $\mathscr{L}(E, D(A))$-valued function associated with $S$ in $\Omega$ by (3.1). Write formula (3.4) for each $u_{\gamma}=$ $S\left(\varphi_{r}\right) v$ (the polynomial in the right-hand side is now dependent on $\gamma$ and will be called $\left.P_{m, p}^{\gamma}\right)$. It follows from (3.5) applied to $u_{\gamma}$ that $A^{m} u_{\gamma} \rightarrow 0$ for all $m$; since $S^{(m n-p)} u_{\gamma} \rightarrow 0$ in $\Omega$ in the sense of distributions, if $\varphi \in \mathscr{D}$ and $\operatorname{supp}(\varphi) \subset \Omega$,

${ }^{5}$ A vector-valued distribution with support in $\{0\}$ may not necessarily be of the form (3.9). 


$$
\int P_{m, p}^{r}(t) \varphi(t) d t \rightarrow 0
$$

But it is not hard to see that (3.11) implies (due to the fact that deg $P_{m, p}^{r}$ is uniformly bounded) that $P_{m, p}^{r} \rightarrow 0$ uniformly on compacts of $R$ together with all its derivatives; using this in (3.4) we obtain the desired result.

\section{The case $n \geqq 3$.}

Theorem 4.1. Let $n$ be an integer $\geqq 3$. Assume that the Cauchy problem for (2.1) is well set and that Assumption (3.1) is satisfied. Then $D(A)=E, A$ is continuous and the series

$$
M_{n}(t A)=\sum_{k=0}^{\infty} \frac{t^{k}}{(n k) !} A^{k}
$$

converges in the topology of $\mathscr{L}(E)$ for all $t>0$. The propagator $S$ of (2.1) is actually a $\mathscr{L}(E)$-valued function given by

$$
S(t)=h(t) \sum_{k=0}^{\infty} \frac{t^{n k+n-1}}{(n k+n-1) !} A^{k} .
$$

Conversely, let the series (4.1) be convergent for all $t>0$. Then the Cauchy problem for (2.1) is well set and the propagator is given by the formula (4.2).

We shall find it necessary to use in the sequel a few facts about analytic functions with values in a quasi-complete barreled locally convex linear topological space $F$. A $F$-valued function $f$ defined in a domain $D \subseteq C$ is analytic in $D$ if the quotient of increments

$$
h^{-1}\{f(z+h)-f(z)\}
$$

has a limit (in the $F$-topology) as $h \rightarrow 0$ for all $z \in D$. (We shall only consider the cases $F=E, F=\mathscr{L}(E)$ ). All the usual properties of scalar-valued functions (Cauchy's formula etc.) can be extended to $F$ valued functions; they can be developed in power series in the usual way, the series being convergent for $\left|z-z_{0}\right|<\rho=\operatorname{dist}\left(z_{0}\right.$, boundary of $D$ ). In general, a power series $\sum a_{n}\left(z-z_{0}\right)^{n}$ with coefficients in $F$ converges absolutely and uniformly in $\left|z-z_{0}\right|<\rho$, diverges in $\left|z-z_{0}\right|>$ $\rho$, where

$$
\rho=\inf \left\{\lim \inf \left|a_{n}\right|^{-(1 / n)} ;|\cdot| \in \mathscr{F}\right\}
$$

(F a set of semi-norms defining the topology of $F$ ). All these simple facts can be proved essentially like in the Banach space case (see [11], Chapter III, §2). If $f(\cdot)$ is an $F$-valued function defined in $D$ and 
such that $\left\langle u^{*}, f\right\rangle$ is a (usual) analytic function for all $u^{*} \in F^{*}$ then $f$ is analytic in the sense outlined above. Likewise, if $B(\cdot)$ is an $\mathscr{L}(F)$ valued function such that $\left\langle u^{*}, B u\right\rangle$ is an ordinary analytic function for all $u^{*} \in F^{*}, u \in F$ then $B(\cdot)$ is analytic as an $\mathscr{L}(F)$-valued function. The proofs of these results also generalize from the Banach space case. ([11], Chapter III, $\S 2$, p. 93); in fact, they are only based in the equicontinuity principle for $\mathscr{L}(F)$ and $F^{*}$ and in quasi-completeness of these spaces.

The preceding "weak" characterizations of vector-valued analytic functions can be used in combination with "scalar" theorems to obtain generalizations to the $F$-valued case. We shall make use of two of such extensions:

(a) if $f$ has first continuous partials in $D$ and satisfies the CauchyRiemann equations with respect to two independent directions then it is analytic

(b) if $f$ is continuous in a domain $D$, analytic in $D$ minus a smooth curve $\Gamma$, then $f$ is actually analytic in all of $D$.

We shall also make use of a slight modification of a result of $\mathrm{L}$. Schwartz (Théorème XXIV of Chapter VI in [10], p. 198).

Auxiliary Lemma 4.2. Let $T \in \mathscr{D}_{0}^{\prime}$. Define for each $\varphi \in \mathscr{D}$, $\varphi_{\xi}(t)=\varphi(t-\xi)$.

Assume that for every $\varphi \in \mathscr{D}_{0}$ the function $\xi \rightarrow T\left(\phi_{\xi}\right), \xi>0$ can be extended to a function analytic in a fixed region containing $\xi>0$. Then $T$ itself coincides with an analytic function in $\xi>0$.

The proof is almost identical to the one for the result of Schwartz. Let $a, b, c, d>0, a<b, c<d$ but otherwise arbitrary, $\mathscr{D}_{[a, b]}=\{\varphi \in \mathscr{D}$; supp $(\varphi) \subseteq[a, b]\}$ with the topology generated by the family of norms $|\varphi|_{p}=\max _{0 \leqq k \leqq p} \max _{a \leqq s \leqq b}\left|\varphi^{(k)}(s)\right|, p=0,1,2, \cdots \mathscr{D}_{[a, b]}^{m}$ the Banach space of all functions $\phi \mathrm{m}$ times continuously differentiable in $R$ with support in $[a, b]$ (norm: $\left.|\cdot|_{m}\right), \mathscr{C}_{[c, d]}$ the space of all continuous functions in $[c, d]$. Let $r>0, B_{r, p}: \mathscr{D}_{[a, b]} \rightarrow \mathscr{C}_{[c, d]}$ defined by

$$
\left(B_{r, p} \varphi\right)(\xi)=\frac{T\left(\varphi_{\xi}\right)^{(p)}}{p ! r^{p}}=\frac{\left(T^{(p)} * I \varphi\right)(\xi)}{p ! r^{p}}
$$

$p=0,1, \cdots$ Reasoning exactly like in [10], p. 198, we see that for some $r$ (depending on $a, b, c, d$ ) the family $\mathscr{B}=\left\{B_{r, p}, p \geqq 0\right\}$ is equicontinuous. Then there exists an integer $m \geqq 0$ and a real number $\varepsilon>0$ such that if $\phi \in \mathscr{D}_{[a, b]},|\varphi|_{m} \leqq \varepsilon$

$$
\left|B_{r, p} \varphi\right|_{0} \leqq 1
$$


in $\mathscr{C}_{[\varepsilon, d]}$. Consequently, $\mathscr{B}$ is as well equicontinuous as a family of operators from $\mathscr{D}_{[a, b]}$-endowed with the $\mathscr{D}^{m}$-topology-to $\mathscr{C}_{[c, d]}$. Let now $\varphi \in \mathscr{D}_{[a, b]}^{m},\left\{\varphi_{k}\right\}$ a sequence in $\mathscr{D}_{[a, b]}$ converging to $\varphi$ in $\mathscr{D}_{[a, b]}^{m}$. It is plain from (4.3) that

$$
B_{r, p}\left(\varphi_{k}\right) \underset{k}{\longrightarrow}\left(p ! r^{p}\right)^{-1} T^{(p)} * I \varphi
$$

in the sense of distributions. On the other hand, if $k$ is large enough, $\left|\varphi_{k}\right|_{m} \leqq|\varphi|_{m}+1$, then

$$
\left|B_{r, p}\left(\varphi_{k}\right)\right|_{0} \leqq \varepsilon^{-1}\left(|\varphi|_{m}+1\right)
$$

in $\mathscr{C}_{[c, d]}$. But $(d / d \xi) B_{r, p}\left(\varphi_{l k}\right)=(p+1) r B_{r, p+1}\left(\varphi_{k}\right)$, thus by virtue of Ascoli's theorem we may assume (passing, if needed, to a subsequence) that for all $p \geqq 1, B_{r, p}\left(\varphi_{k}\right)$ is convergent in $\mathscr{D}_{[c, d]}^{0}$. Then each distribution $\left(p ! r^{p}\right)^{-1} T^{(p)} * I \varphi$ coincides in $(c, d)$ with a continuous function and by virtue of the estimates (4.5) the set of all these functions is uniformly bounded in $[c, d]$. This shows, via the definition of $B_{r, p}$ that $T * I \varphi$ is actually analytic in a neighborhood of $[c, d]$ for any $\phi \in \mathscr{D}_{[a, b]}^{m}$.

Finally, let $Y_{m+2}$ the distribution in $\S 2, \chi$ a function in $\mathscr{D}$ such that $\chi=1$ in $|t| \leqq(b-a) / 4, \chi=0$ in $|t|>(b-a) / 3, k=(a+b) / 2$. Plainly $\varphi(t)=\left(\chi Y_{m+2}\right)(t-k)$ belongs to $\mathscr{D}_{[a, b]}^{m}$, while $\varphi^{(m+2)}=\tau_{k} \delta+\eta, \tau_{k}$ the operator of translation by $k, \eta \in \mathscr{D}$.

We have

$$
(-1)^{m+2}(T * I \phi)^{(m+2)}-T * I \eta=\tau_{-k} T .
$$

Since the left side of the preceding inequality is analytic, so is $T$ in $[c+k, d+k]$; since $k=(a+b) / 2$ can be arbitrarily small and $c, d$ are unrestricted, the result follows.

Proof of Theorem 4.1. Let $\omega=\exp (2 \pi i / n), C_{k}=\{\zeta \in C ; 2 k \pi i / n \leqq$ $\arg \zeta \leqq 2(k+1) \pi i / n\}, k=0,1, \cdots, n-1$. Let $\varphi$ be a fixed element in $\mathscr{D}_{0}, u \in E$. Define $E$-valued functions $g_{0}$ (in $C_{0}$ ), $g_{n-1}$ (in $C_{n-1}$ ) as follows:

$$
\begin{aligned}
& g_{1}(\xi+\eta \omega)=\omega^{-1} \sum_{j=0}^{n-1} \omega^{-j} G^{(j)}(\eta) S^{(n-1-j)}\left(\varphi_{\xi}\right) u, \\
& g_{2}\left(\xi+\eta \omega^{-1}\right)=\omega \sum_{j=0}^{n-1} \omega^{j} G^{(j)}(\eta) S^{(n-1-j)}\left(\varphi_{\xi}\right) u .
\end{aligned}
$$

If $\xi, \xi^{\prime}, \eta, \eta^{\prime} \geqq 0$ we have, for any two integers $p, q \geqq 0$

$$
\begin{aligned}
G^{(p)}\left(\eta^{\prime}\right) S^{(q)}\left(\varphi_{\xi^{\prime}}\right) u & -G^{(p)}(\eta) S^{(q)}\left(\varphi_{\xi}\right) u=G^{(p)}\left(\eta^{\prime}\right)\left(S^{(\varphi)}\left(\varphi_{\xi^{\prime}}\right)\right. \\
& \left.-S^{(q)}\left(\varphi_{\xi}\right)\right) u+\left(G^{(p)}\left(\eta^{\prime}\right)-G^{(p)}(\eta)\right) S^{(q)}\left(\varphi_{\xi}\right) u .
\end{aligned}
$$

This and the regularity results in $\S 3$ show that $g_{0}\left(g_{n-1}\right)$ has continu- 
ous partials of any order in $C_{0}\left(C_{n-1}\right)$. We compute now the first partials. We have

$$
\begin{aligned}
& \frac{\partial}{\partial \eta} g_{0}=\sum_{j=1}^{n} \omega^{-j} G^{(j)}(\eta) S^{(n-j)}\left(\varphi_{\xi}\right) u \\
& \frac{\partial}{\partial \xi} g_{0}=\omega^{-1} \sum_{j=0}^{n-1} \omega^{-j} G^{(j)}(\eta) S^{(n-j)}\left(\varphi_{\xi}\right) u .
\end{aligned}
$$

Consequently

$$
\left(\frac{\partial}{\partial \eta}-\omega \frac{\partial}{\partial \xi}\right) g_{0}=G^{(n)}(\eta) S\left(\varphi_{\xi}\right) u-G(\eta) S^{(n)}\left(\varphi_{\xi}\right) u .
$$

Since $G^{(n)}(\eta) S\left(\varphi_{\xi}\right) u=A G(\eta) S\left(\varphi_{\xi}\right) u=G(\eta) A S\left(\varphi_{\xi}\right) u=G(\eta) S^{(n)}\left(\varphi_{\xi}\right) u$ the right-hand side of (4.4) vanishes. But then (4.8) reduces to the Cauchy-Riemann equation for $g_{0}$ (with respect to the directions $1, \omega$ ) and consequently $g_{0}$ is holomorphic in $C_{0}^{0}$, the interior of $C_{0}$. Proceeding in exactly the same way with $g_{n-1}$ in $C_{n-1}$ we obtain the equation

$$
\left(\frac{\partial}{\partial \eta}-\omega^{-1} \frac{\partial}{\partial \xi}\right) g_{2}=0
$$

which likewise implies that $g_{n-1}$ is holomorphic in $C_{n-1}$. By virtue of Corollary 3.3,

$$
g_{0}(\xi)=g_{n-1}(\xi)=S\left(\varphi_{\xi}\right) u .
$$

This shows that the function $g$ defined as $g_{0}$ in $C_{0}, g_{n-1}$ in $C_{n-1}$ is continuous in $C_{0} \cup C_{n-1}$, and thus analytic there.

Denote now $\mathscr{L}_{S}(E)$ the space of all linear continuous operators from $E$ to $E$ with the topology of simple convergence (or strong topology; see [2], Chapter III, §3). It follows from the BanachSteinhaus theorem that $\mathscr{L}_{S}(E)$ is quasi-complete ([2], Chapter III, $\S 3$ ). Moreover, any continuous linear functional in $\mathscr{E}_{S}(E)$ can be written

$$
A \longrightarrow \sum_{k=1}^{m}\left\langle u_{k}^{*}, A u_{k}\right\rangle
$$

where $u_{1}, \cdots, u_{m} \in E, u_{1}^{*}, \cdots, u_{m}^{*} \in E$ ([2], Chapter IV, §2, Proposition 11). Consider the propagator $S$-as we may-as an element of $\mathscr{D}^{\prime}\left(\mathscr{L}_{S}(E)\right)$ or, rather, as an element of $\mathscr{D}_{0}^{\prime}\left(\mathscr{L}_{S}(E)\right)=\mathscr{L}\left(\mathscr{D}_{0} ; \mathscr{L}_{S}(E)\right)$. It follows from our results on the function $g$ defined by (4.6), (4.7) and from the equality (4.9) that the distributions in $\mathscr{D}_{0}^{\prime}$ obtained from $S$ by applying arbitrary elements of $\left(\mathscr{L}_{S}(E)\right)^{*}$ coincide with functions analytic in $\xi>0$ in particular, with $C^{\infty}$ functions. Applying a result in [8], p. 55 (the change of $\mathscr{D}$ by $\mathscr{D}_{0}$ has no particular significance), we see that $S$ itself coincides in $t>0$ with a $\mathscr{L}(E)$-valued function 
$G(\cdot)$ infinitely differentiable in the $\mathscr{L}_{S}(E)$-topology, that is $G(\cdot) u$ is a $C^{\infty}$ function in $\xi>0$ for all $u \in E$. Clearly if $u \in D=D\left(A^{\infty}\right)$ then $G u$ coincides with the function defined in $\S 3$. We now extend $G$ to the complex plane as follows; if $\zeta=\xi \omega^{k}+\eta \omega^{k+1} \in C_{k}$

$$
G\left(\xi \omega^{k}+\eta \omega^{k+1}\right) u=\omega^{-(k+1)} \sum_{j=0}^{n-1} \omega^{-j} G^{(j)}(\eta) G^{(n-1-j)}(\xi) u,
$$

$0 \leqq k \leqq n-1$. It follows from the equicontinuity principle $(\S 2)$ that the family $\{G(t) ; t \in e\}$, $e$ any compact subset of $(0, \infty)$ is equicontinuous in $\mathscr{L}(E)(\{G(t) u ; t \in e\}$ is bounded in $E$ for any $u \in E)$. This, and the fact that $G$ is strongly $C^{\infty}$ in $\xi>0$ shows that $G(\cdot)$ as defined by (4.10) has any number of continuous partials in $C_{k}^{0}$, the interior of $C_{k}$ for any $k=0, \cdots, n-1$. An argument similar to the one for the function $g$ shows that $G u$ satisfies in each $C_{k}^{0}$ the Cauchy-Riemann equations, and is thus analytic; a fortiori, $G$ itself, as a $\mathscr{L}(E)$-valued function is analytic in $C_{0}^{0} \cup \cdots \cup C_{n-1}^{0}$.

We now examine more carefully the equality (4.10) when $u \in D$. Let $\left\{\varphi_{n}\right\}$ be a "smoothing kernel" in $\mathscr{D}$, i.e., let $\varphi_{n} \geqq 0, \int \varphi_{n} d t=1$, $\operatorname{supp}\left(\varphi_{n}\right) \rightarrow 0$ as $n \rightarrow \infty$. If $\xi, \eta>0$ we have, in view of Lemma 2.3, $S\left(\left(\varphi_{n}\right)_{\xi}\right) S\left(\left(\varphi_{m}\right)_{\eta}\right) u=S\left(\left(\varphi_{m}\right)_{\eta_{i}}\right) S\left(\left(\varphi_{n}\right)_{\xi}\right) u$; letting $m, n \rightarrow \infty$ we obtain

$$
G(\xi) G(\eta)=G(\eta) G(\xi)
$$

Differentiating the relation (4.11) and making use of the new equalities thus obtained in (4.10), we easily see (by applying the fact that $G(\xi) u, u \in D$ is smooth in $\xi \geqq 0)$ that $G(\zeta) u, \zeta \in C_{k}$ is actually continuous even if $\xi$ or $\eta$ are zero; thus $G(\zeta) u$ is continuous in $C_{k}$, except perhaps at the origin. On the other hand, it is not difficult to see by using Corollary 3.3 that the different definitions of $G$ match at the divisory rays $\xi \omega^{k}, \xi \geqq 0, k=0, \cdots, n-1$. Consequently $G(\cdot) u$ is continuous in all of $C$-except perhaps at the origin; being holomorphic in $C_{0}^{0} \cup \cdots \cup C_{n-1}^{0}$, it is actually holomorphic in all of $C$-again, with the possible exception of the origin.

We apply now the same "regularization" method used to obtain (4.11) to the equation (2.4). We obtain

$$
G(s+t)=\sum_{j=1,}^{n-1} G^{(j)}(s) G^{(n-1-j)}(t)
$$

for $s, t>0$. Applying both sides of this equality to a $u \in D$ and using analiticity of $G u$, we can extend (4.12) to all complex $z, \zeta \in C \backslash\{0\}$ as long as $z, \zeta$ do not both belong to a divisory ray; in particular

$$
G(z+\zeta) u=\sum_{j=0}^{n-1} G^{(j)}(z) G^{(n-1-j)}(\zeta) u
$$


if $z, \zeta \in \bigcup_{k=0}^{n-1} C_{k}^{0}$. But for these values of $z, \zeta$ the operators in the right-hand side of (4.13) are continuous, and then (4.13) can be extended to all $u \in E$. Observe finally that (4.13) allows us to express $G u$ near a divisory ray (or near the origin) in a linear and continuous way by means of its values away from them, thus $G u$ is actually holomorphic in $C$ for any $u \in F$; a fortiori, $G$ is a $\mathscr{L}(E)$-valued entire function.

We compute now the coefficients in the Maclaurin series of $G$. According to Corollary $3.3 G^{(2 n-1)}(0) u=A u$ for $u \in D$; since $A$ is closed and $D$ dense, $D(A)=E$ and $A=G^{(2 n-1)}(0)$ is continuous. The fact that $G^{(k)}(0)=0$ if $k \neq m n-1, G^{(m n-1)}(0)=A^{m-1}$ for $m \geqq 1$, can be proved by using Corollary 3.3 and the denseness of $D$. Then

$$
G(\zeta)=\sum_{k=0}^{\infty} \frac{\zeta^{n k+n-1}}{(n k+n-1) !} A^{k} .
$$

The convergence of the series for $M_{n}(t A)$ is clear, as $M_{n}(t A)=$ $G^{(n-1)}\left(t^{1 / n}\right)$. The final step of the proof of the direct part of Theorem 4.1 (that is, to show that $S=h G$ ) will be left to the next Remark 4.4 and consists in showing directly that $(h G)^{(n)}-A(h G)=\delta \otimes I$; by uniqueness, it follows that $(h G) u=S u$ for all $u \in E$ and then $h G=S$.

REMARK 4.3. The proof of Theorem 4.1 depends crucially on the fact that the $n^{t h}$ roots of unity span $C$, thought of as a real vector space or, more precisely, on the possibility of writing any $\zeta \in C$ in the form $\zeta=\xi \omega^{k}+\eta \omega^{k+1}$ for some integer $k, \xi, \eta \geqq 0, \omega=\exp (2 \pi i / n)$. This is obviously true only if $n \geqq 3$; for if $n=1, \omega=1$; if $n=2$, $\omega=-1$.

REMARK 4.4. We end the proof of Theorem 4.1 by establishing the following slightly more general form of its converse part.

LemmA 4.5. Let $\alpha>0$ (not necessarily an integer). Assume $A \in \mathscr{L}(E)$ and that

$$
M_{\alpha}(t A) u=\sum_{k=0}^{\infty} \frac{t^{k}}{\Gamma(\alpha k+1)} A^{k} u^{6}
$$

converges for all $t>0$ in $E$ for each $u \in E$. Then the Cauchy problem for

$$
U^{(\alpha)}-A U=T
$$

is well set; the propagator of (4.14) is a $C^{\infty}, \mathscr{L}(E)$-valued function in $t \geqq 0$ given by

${ }^{1} M_{\alpha}(t)$ is the Mittag-Leffler function of classical analysis. 


$$
S(t)=h(t) \sum_{k=0}^{\infty} \frac{t^{\alpha k+\alpha-1}}{\Gamma(\alpha k+\alpha)} A^{k},
$$

the series (4.15) being convergent in $\mathscr{L}(E)$ for all $t$.

Proof. Since the series defining $M_{\alpha}(t A) u$ converges for all $t$, the same is true of the series defining $M_{\alpha}(\zeta A) u, \zeta \in C$. By virtue of the Banach-Steinhaus theorem ([2], Chapter III, $\S 3 M_{a}(\zeta A)$ is a $\mathscr{L}(E)$ valued function; since $M_{\alpha}(\zeta A) u$ is analytic for each $u \in E$, the same is true of $M_{\alpha}(\zeta A)$ as a function with values in $\mathscr{L}(E)$. This is easily seen to imply convergence of the series in (4.15) in the topology of $\mathscr{L}(E)$ for all $t$, uniformly on compact subsets of $R$ (and thus in $\left.\mathscr{D}^{\prime}(\mathscr{L}(E))\right)$. Recall now that, for $\beta>0$ the distribution $Y_{\beta} \in \mathscr{D}^{\prime}$ used in $\S 2$ to define fractional derivatives coincides with the function $(h(t) t)^{\beta-1} / \Gamma(\beta)$. Then

$$
\begin{aligned}
S^{(\alpha)} & =Y_{-\alpha^{*}}\left(\sum_{k=0}^{\infty} Y_{\alpha(k+1)} \otimes A^{k}\right) \\
& =\sum_{k=1}^{\infty} Y_{\alpha k} \otimes A^{k}+Y_{0} \otimes I=A S+\delta \otimes I .
\end{aligned}
$$

Consequently $S$ satisfies the equation (2.3) and this implies that $U=$ $S * T$ satisfies the equation $(2.1)$ for any $T \in \overline{\mathscr{D}}_{+}^{\prime}(E)$. It only remains, then, the question of uniqueness, which we can verify in the form $\left(b^{\prime}\right), \S 2$. Let $u(\cdot)$ be a $E$-valued $C^{\infty}$ function, null for $t \leqq 0$ and such that

$$
u^{(\alpha)}(t)=A u(t)
$$

for $t \leqq a, a>0$. Take the convolution product of both sides of (4.16) with the (function) $Y_{\alpha}$; we obtain $u(t)=A\left(Y_{\alpha} * u\right)(t)$ for $t \leqq a$. Iterating this equality $m$ times $w$ get $u(t)=A^{m}\left(Y_{m \alpha} * u\right)(t)$ for $t \leqq a$, or

$$
u(t)=\frac{1}{\Gamma(m \alpha)} \int_{0}^{t}(t-s)^{m \alpha-1} A^{m} u(s) d s
$$

for $t \leqq a$. Observe now that $\{u(s), 0 \leqq s \leqq t\}$ is a bounded set in $E$ for every $t>0$, then as a consequence of the definition of the topology of $\mathscr{L}(E)$ and of the fact that the series for $M_{\alpha}(t A)$ has infinite radius of convergence in $\mathscr{L}(E)$, if $|\cdot| \in \mathscr{E}$

$$
\lim _{m \rightarrow \infty}\left|\frac{(t-s)^{m \alpha-1}}{\Gamma(m \alpha)} A^{m} u(s)\right|=0
$$

uniformly for $0 \leqq s \leqq t$. Applying this estimate in the integral (4.17) we get $u(t)=0$ for all $t<a$. This result, after a clearly permissible translation is equivalent to $\left(b^{\prime}\right)$ of $\S 2$. 
REMARK 4.6. In the case $E$ is a Banach space, J. Chazarain ([12] and personal communication) has characterized the operators $A$ for which the Cauchy problem for (4.14) is well set for any $\alpha, 0<\alpha<\infty$ not necessarily an integer in terms of the location of $\sigma(A)$, the spectrum of $A$ and the growth of $R(\lambda ; A)=(\lambda I-A)^{-1}$. In particular, if $\alpha>2$ the Cauchy problem for (4.14) is well set if and only if $A$ is everywhere defined and bounded. This result, as well as the one in the next section suggest that Theorem 4.1 is probably true for all $\alpha>2$, i.e., that every time the Cauchy problem for (4.14) is well set for $\alpha>2$ we have $D(A)=E, A$ is continuous and the series for $M_{\alpha}(t A)$ is convergent for all $t>0$. However, the method used here, that is to exploit the simple functional equation (2.4) to extend $S$ to the complex plane breaks down when $\alpha$ is not an integer. Finally, note that Theorem 4.1 generalizes Theorem 3.1 of [3] but apparently only in the case the Cauchy problem for $u^{(n)}=A u$ is, in the terminology of [3] uniformly well posed in $t \geqq 0$ (see [12] for a proof). For if the Cauchy problem for $u^{(n)}=A u$ is only well posed in $t>0$ the propagator $S_{n-1}$ which plays the role of $S$ in [3] may a priori grow arbitrarily fast as $t \rightarrow 0$ and then does not define a distribution in any obvious way. It is not difficult, however, to include also this case in our results. In fact let $S$ be a distribution in $\mathscr{D}_{0}^{\prime}(\mathscr{L}(E, D(A))$ satisfying Equation (2.4); if the regularity results of $\S 2$ are postulated (they can be easily seen to hold in the situation of [3]) then the proof of Theorem 4.1 can be carried out just in the same way and its conclusion holds. As for Equation (2.4), it is an immediate consequence of Equation (2.8) of [3].

5. Exponential increase of $S$. We relax in this section the requirement that $\alpha$ be an integer, but we are then forced to impose restrictions on the growth of $S$ at $\infty$.

A few simple properties of vector-valued Laplace transforms will be used in the sequel. Denote, as usual, by $\mathscr{S}$ the space of all infinitely differentiable, complex-valued functions $\varphi$ that decrease at $|\infty|$ faster than any power of $1 /|t|$ together with all their derivatives, endowed with its usual Schwartz topology ([10], Chapter VII, p. 234). The space $\mathscr{S}^{\prime}(F)$ (of "tempered", $F$-valued distributions) is $\mathscr{L}(\mathscr{S} ; F)$. Given $\omega \in R, 0<\omega<\infty$ we write $\Gamma_{\omega}=(\omega, \infty)$; the space $\left(\mathscr{S}^{\prime}\left(\Gamma_{\omega}\right)\right)(F)$ consists of all distributions $T \in \mathscr{D}^{\prime}(F)$ such that $e_{\lambda} T \in \mathscr{S}^{\prime}(F)$ for all $\lambda \in \Gamma_{\omega}, e_{\lambda}$ the $C^{\infty}$ function defined by $e_{\lambda}(t)=e^{-\lambda t}$. Any distribution $T \in\left(\mathscr{S}^{\prime}\left(\Gamma_{\omega}\right)\right)(F)$ has a Laplace transform

$$
2 T=(\Omega T)(\lambda), \lambda=\xi+i \eta,
$$

a $F$-valued function holomorphic in $\operatorname{Re} \lambda>\omega([8]$, p. 74). If, in ad- 
dition, $T=0$ for $t<0$ then for all $a>\omega, u^{*} \in F^{*}$ there exists a polynomial $p$ such that

$$
\left|\left\langle(\Omega T)(\lambda), u^{*}\right\rangle\right| \leqq p(|\lambda|)
$$

for $\operatorname{Re} \lambda \geqq a$ (this is an easy consequence of the "scalar-valued" theorem; see [10], Chapter VIII, p. 310, and [6]) where the polynomial may depend on $u^{*}$. Conversely, if $L$ is a $F$-valued function holomorphic in $\operatorname{Re} \lambda>\omega$ and such that estimates of the form (5.1) hold for it, then $L=\& T$ where $T$ is a (unique) distribution in $\left(\mathscr{S}^{\prime}\left(\Gamma_{\omega}\right)\right)(F)$, $T=0$ for $t<0$ (see again [8], p. 74, and [10], p. 310). If $T=f, f$ an ordinary $F$-valued function (say, continuous, zero for $t<0$ and such that $\left\{e_{\lambda}(t) f(t) ; t \geqq 0\right\}$ is bounded in $F$ for any $\left.\lambda \in \Gamma_{\text {(u) }}\right)$ then $\mathbb{Q} T$ coincides with its ordinary Laplace transform, that is

$$
(\Omega T)(\lambda)=\int_{0}^{\infty} e^{-\lambda t} f(t) d t .
$$

Finally, let $T \in\left(\mathscr{S}^{\prime}\left(\Gamma_{\omega t}\right)\right)(F), V \in \mathscr{S}^{\prime}\left(\Gamma_{\omega}\right)$ (and assume, for the sake of simplicity, that both $V, T$ are zero for $t<0)$. Then the Laplace transform of the convolution $V * T \in\left(\mathscr{S}^{\prime}\left(\Gamma_{\omega}\right)\right)(F)$ is

$$
\mathfrak{L}(V * T)=\mathfrak{\&} V \mathbb{2} T
$$

([9], Proposition 43, p. 186). We shall only use this result for $V=$ $Y_{\beta}=\left(\mathrm{Pf} . t^{\beta-1}\right) / \Gamma(\beta) ; Y_{\beta} \in \mathscr{S}^{\prime}$ for all $\beta$ and its Laplace transform equals $\mathcal{L}\left(Y_{\beta}\right)(\lambda)=\lambda^{-\beta}$; by virtue of the preceding observation,

$$
\mathfrak{R}\left(T^{(\kappa)}\right)(\lambda)=\mathfrak{R}\left(Y_{-\alpha} * T\right)(\lambda)=\lambda^{\alpha} \mathfrak{R}(T)(\lambda) .
$$

We shall find it useful to introduce at this point a new space of distributions. We call $\left(\mathscr{S}_{f}^{\prime}\left(\Gamma_{\omega}\right)\right)(F)$ the set of all distributions in $\left(\mathscr{S}^{\prime}\left(\Gamma_{\omega}\right)\right)(F)$ such that, for any $\lambda \in \Gamma_{\omega}$

$$
e_{\lambda} T=f^{(m)}
$$

for some $m \geqq 0$, where $f$ is a continuous function defined in $R$, with values in $F$ and such that

$$
\left\{(1+|t|)^{-p} f(t) ; t \in R\right\}
$$

is a bounded set in $F$ for some $p \geqq 0$. (Note that any $T \in \mathscr{D}^{\prime}(F)$ that satisfies the preceding condition belongs to $\left.\left(\mathscr{S}^{\prime}\left(\Gamma_{\omega}\right)\right)\left(F^{\prime}\right)\right)$. A characterization of some elements in $\left(\mathscr{S}_{f}^{\prime}\left(\Gamma_{\omega}\right)\right)(F)$ is given by the following

Auxiliary Lemma 5.1. Let $T \in\left(\mathscr{S}^{\prime}\left(\Gamma_{\omega}\right)\right)(F)$. Then $T \in\left(\mathscr{S}_{f}^{\prime}\left(\Gamma_{\omega}\right)\right)(F)$ and has support in $t \geqq 0$ if and only if for each $a>\omega$ there exists a polynomial $p \geqq 0$ such that the set 


$$
\left\{(1+p(|\lambda|))^{-1}(\Omega T)(\lambda) ; \operatorname{Re} \lambda \geqq a\right\}
$$

is bounded in $F$.

Proof. Observe first that the function $f$ in (5.2) can be assumed to be zero for $t<0$. For if $h$ is the Heaviside function,

$$
e_{\lambda} T=h e_{\lambda} T=h f^{(m)}=(h f)^{(m)}-\sum_{j=1}^{m-1} \delta^{(j)} \otimes f^{(m-1-j)}(0) .
$$

Consequently $g=h f-\sum_{j=1}^{m-1} Y_{m-j} f^{(m-1-j)}(0)$ (which is zero in $t<0$ ) has the same $m$-th derivative as $f$. If $g$ is not continuous, replace $m$ by $m+1, g$ by $\int_{0}^{t} g(s) d s$.

We now use (5.2) for $\lambda=a^{\prime}, \omega<a^{\prime}<a$, and the relation

$$
(\& T)(\lambda)=\left(\Re\left(e_{a^{\prime}} T\right)\right)\left(\lambda-a^{\prime}\right) . \quad \text { Since }
$$

$\left(\mathcal{L}\left(e_{a^{\prime}} T\right)\right)(\lambda)=\left(\mathcal{L}\left(f^{(m)}\right)\right)(\lambda)=\lambda^{m}(\mathcal{R} f)(\lambda)$ and, on the other hand

$$
|(\& f)(\lambda)| \leqq \frac{K}{\operatorname{Re} \lambda-\varepsilon}, \operatorname{Re} \lambda \geqq \varepsilon
$$

for any continuous semi-norm $|\cdot|$ in $F$ and any $\varepsilon>0$ (the constant $K$ may depend on $|\cdot|, \varepsilon)$ the result follows. Conversely, assume that (5.4) is bounded for all $a>\omega, p$ the polynomial corresponding to $a$, $m=$ degree of $p, \omega<a^{\prime}<a$. Define

$$
g(t)=\frac{1}{2 \pi i} \int_{\operatorname{Re} \lambda=a^{\prime}} \lambda^{-(m+2)}(\Omega T)(\lambda) e^{\lambda t} d t
$$

for $t \in R$. It is not difficult to see that $g$ is a continuous function, zero for $t \leqq 0$, that the set $\left\{e^{-a^{\prime} t} f(t) ; t \in R\right\}$ is bounded in $F$ and that

$$
(\Omega f)(\lambda)=\lambda^{-(m+2)}(\Omega T)(\lambda)
$$

in $\operatorname{Re} \lambda>a^{\prime}$. Equality (5.5) and uniqueness of Laplace transforms plainly imply

$$
T=f^{(m+2)}
$$

Observe, finally, that

$$
e_{a} T=e_{a}\left(e_{-a}\left(e_{a} f\right)\right)^{(m+2)}=\sum_{j=0}^{m+2}\left(\begin{array}{c}
m+2 \\
j
\end{array}\right) a^{m-2-j}\left(e_{a} f\right)^{(j)}
$$

which ends the proof.

Theorem 5.2. Let $\alpha>2$. Assume the Cauchy problem for the equation 


$$
U^{(\alpha)}-A U=T
$$

is well set and that the propagator $S$ belongs to the space

$$
\left.\left(\mathscr{S}_{f}^{\prime} \Gamma_{\omega}\right)\right)(\mathscr{L}(E, D(A))
$$

for some $\omega, 0 \leqq \omega<\infty$. Then $A$ is continuous, $D(A)=E, R(\lambda ; A)=$ $(\lambda I-A)^{-1}$ exists for $|\lambda|$ large enough and the function $R(\cdot ; A)$ is analytic at $\infty$. Conversely, the preceding conditions imply that the Cauchy problem for (5.6) is well set and that $S \in\left(\mathscr{S}_{f}^{\prime}\left(\Gamma_{\omega}\right)\right)(\mathscr{L}(E))$ for some $\omega<\infty$.

Proof. Taking Laplace transforms of both sides of the equation (2.3) (that is, $Y_{-\alpha} * S-A S=\delta \otimes I$ ) satisfied by the propagator we obtain

$$
\left(\lambda^{\alpha} I-A\right)(2 S)(\lambda)=I
$$

for $\operatorname{Re} \lambda>\omega$, where $(\mathcal{R} S)(\lambda)$ is a $\mathscr{P}(E, D(A))$-valued holomorphic function. By virtue of Lemma 2.3 if $u \in D(A)$ we also have the equality

$$
Y_{-\alpha} *(S u)-S(A u)=\delta \otimes u
$$

thus

$$
(\mathcal{R} S)(\lambda)\left(\lambda^{\alpha} I-A\right) u=u
$$

as well. But equalities (5.7), (5.8) plainly imply that $R\left(\lambda^{\alpha} ; A\right)$ exists and equals $(\& S)(\lambda)$ for $\operatorname{Re} \lambda>\omega$. Since $\left\{\mu \in C ; \mu=\lambda^{\alpha}\right.$, $\left.\operatorname{Re} \lambda>\omega\right\}$ contains a neighborhood of $\infty$ if $\alpha>2$ (more precisely, the region $|\lambda|>$ $\left.r=\left(\omega^{2}+\tau^{2}\right)^{\alpha / 2}, \tau=\omega t g(\pi / \alpha)\right), R(\lambda ; A)$ exists for $|\lambda|$ large. We develop now $R(\cdot ; A)$ in Laurent series around $\infty$,

$$
R(\lambda ; A)=\sum_{j=1}^{\infty} \lambda^{-j} D_{j}+D_{0}+\sum_{j=1}^{\infty} \lambda^{j} K_{j}
$$

where $D_{j}, K_{j}$ are elements of $\mathscr{P}(E, D(A))$. Using the relation

$$
(\lambda I-A) R(\lambda ; A)=I
$$

in (5.9) and equating coefficients in the series so obtained we get the system of equations

$$
\begin{aligned}
D_{j+1} & =A D_{j}, j \geqq 1 \\
D_{1} & =A D_{0}+I \\
D_{0} & =A K_{1} \\
K_{j} & =A K_{j+1}, j \geqq 1 .
\end{aligned}
$$

Applying now Lemma 5.1 we see that $R(\lambda ; A)=(R S)\left(\lambda^{1 / \alpha}\right)$ increases 
at $\infty$ less than a polynomial, and then $K_{m}=0$ for some $m \geqq 1$; using equations (5.10), we get $K_{m-1}=\cdots=K_{1}=0, D_{0}=0, D_{1}=I$ and consequently $A=D_{2}$; this shows that $A$ is continuous and that $R(\cdot ; A)$ is analytic at $\infty$ as claimed.

Assume now that $A$ is continuous and that $R(\lambda ; A)$ exists in a neighborhood of $\infty$ and is analytic there. Since the development of $R(\cdot ; A)$ at $\infty$ is

$$
R(\lambda ; A)=\sum_{j=0}^{\infty} \lambda^{-(j+1)} A^{j},|\lambda|>r
$$

we see that if $|\cdot|$ is a continuous semi-norm in $\mathscr{f}(E), \varepsilon>0$

$$
\left|A^{j}\right| \leqq K(r+\varepsilon)^{j}, j \geqq 1
$$

for some $K<\infty$. But then the conditions of Lemma 4.5 are satisfied and consequently the Cauchy problem for (5.6) is well set. We now estimate the propagator $S$. By virtue of (5.11) and of the formula (4.15),

$$
|S(t)| \leqq K \sum_{k=0}^{\infty} \frac{t^{\alpha k+\alpha-1}(r+\varepsilon)^{k}}{\Gamma(\alpha k+\alpha)} .
$$

It follows from results in [5], Chapters IV, V, and VI on asymptotic estimates at $m$ of Maclaurin series that

$$
\sum_{k=0}^{\infty} \frac{t^{\alpha(k+1)-1}}{\Gamma(\alpha k+\alpha)}=-\frac{1}{\alpha} e^{t}(1+o(1))
$$

as $t \rightarrow m$, thus

$$
|S(t)| \leqq K^{\prime} \exp \left((r+\varepsilon)^{1 / \alpha} t\right)
$$

for $t \geqq 0$. This shows that $S \in\left(\mathscr{S}_{f}^{\prime}\left(\Gamma_{m}\right)\right)(\mathscr{C}(E))$ for $\omega=r^{1 / \alpha}$ and therefore ends the proof.

A number of comments are in order. If $F$ is a Banach space and $T \in\left(\mathscr{S}^{\prime}\left(\Gamma_{\omega}\right)\right)(F)$, then $T \in\left(\mathscr{S}_{f}^{\prime}\left(\Gamma_{\omega}\right)\right)(F)$; the reason being that, since $F^{*}$ is a Banach and then a Baire space, a category argument allows us to pass from the "pointwise" estimates (5.1) for $2 T$ to the "uniform" estimate in Lemma 5.1. Thus we can change $\left(\mathscr{S}_{f}^{\prime}\left(\Gamma_{\omega}\right)\right)(\mathscr{P}(E, D(A))$ by $\left(\mathscr{S}^{\prime}\left(\Gamma_{\omega}\right)\right)(\mathscr{P}(E, D(A))$ in the statement of Theorem 5.2. In the general case these two spaces may be different, and we do not know whether the change is possible, i.e., whether or not $S$ has to be assumed to have "finite order" globally.

Consider the conditions $\left(1_{\alpha}\right) A$ is continuous and the series defining $M_{\alpha}(t A)$ converges for all $t$. (2) $R(\lambda ; A)$ exists for large $|\lambda|$ and is analytic at $\infty$. If $E$ is a Banach space, $\left(1_{\alpha}\right)$ for any $\alpha, 0<\alpha<\infty$ 
and (2) are equivalent to the fact that $A$ is bounded. In the general case we can only say that $1_{\alpha} \Rightarrow 1_{\beta}$ if $\alpha \leqq \beta, 2 \Rightarrow 1_{\alpha}, 0<\alpha<\infty$. The reverse implications are in general false, as we shall now see.

Let $E$ be the space of all functions $x \rightarrow u(x)$ defined and continuous in $x \geqq 0$ and such that

$$
|u|_{n}=\sup _{x \geqq 0}\left|u(x) e^{n x}\right|<\infty,
$$

$n=0,1, \cdots$. If we assign to $E$ the topology generated by the family $\mathscr{E}=\left\{|\cdot|_{0}, \cdots\right\}$ of semi-norms $E$ becomes a Fréchet space. If $0<\beta<\infty$ and we define

$$
\left(A_{\beta} u\right)(x)=x^{\beta} u(x)
$$

then $A_{\beta}$ is a continuous operator in $E$. In order to compute $M_{\alpha}\left(t A_{\beta}\right)$ we use the following asymptotic estimate for the Mittag-Leffler function $M_{\alpha}$,

$$
M\left(t^{\alpha}\right)=\frac{1}{\alpha} e^{t}(1+o(1))
$$

(see again [5], Chapter VI). Let now $t$ be fixed, $\alpha \geqq \beta, j<k$. The operator $\sum_{p=j}^{k}\left(t A_{\beta}\right)^{p} / \Gamma(\alpha p+1)$ coincides with the operator of multiplication by

$$
r_{j, k}(x)=\sum_{p=j}^{k}\left(t x^{\beta}\right)^{p} / \Gamma(\alpha p+1) .
$$

Then if $u \in E, n \geqq 0$

$$
\left|r_{j, k} u\right|_{n} \leqq\left(\max _{x>0}\left|r_{j, k}(x)\right| e^{-2 x}\right)|u|_{n+2} .
$$

Now, by virtue of (5.12)

$$
\left|\boldsymbol{r}_{j, k}(x) e^{-2 x}\right| \leqq M_{\alpha}\left(t x^{\beta}\right) e^{-2 x} \leqq K \exp \left(x^{\beta / \alpha}-2 x\right)
$$

for $x \geqq 0, K$ independent of $j, k$. Since, on the other hand, $\lim _{j, k \rightarrow \infty} r_{j, k}(x)=0$ uniformly on compacts of $x \geqq 0$, it is clear that the series for $M_{\alpha}\left(t A_{\beta}\right)$ converges for all $t$ to the operator of multiplication by $M_{\alpha}\left(t x^{\beta}\right)$. But if we assume that $\alpha<\beta$ and the series for $M_{\alpha}\left(t A_{\beta}\right)$ is convergent, then the limit has also to be the operator of multiplication by $M_{\alpha}\left(t x^{\beta}\right)$; but, by virtue of (5.12), this operator is not continuous in $E$ (the operator of multiplication by $\exp \left(x^{\gamma}\right), \gamma>1$ is not continuous in $E$ ). Consequently $A_{\beta}$ satisfies $1_{\alpha}$ for $\alpha \geqq \beta$ but not for $\alpha<\beta$. It is not difficult to construct an operator satisfying $1_{\alpha}$ for any $\alpha>0$ but not 2 ; in fact, let

$$
(A u)(x)=\log (1+x) u(x) .
$$


By small modifications of the reasoning above it can be shown that $M_{\alpha}(t A)$ converges for all $\alpha>0, t \in R$. But $\sigma(A)$ coincides with the positive real axis, then 2 is violated. Applying the results of Sections 4 and 5 we see that the Cauchy problem for

$$
U^{(\alpha)}-A_{\beta} U=T
$$

is well set for $\alpha \geqq \beta$ (the propagators increase at $\infty$ faster than any exponential), is not well set if $2<\alpha<\beta$, at least if $\alpha$ is an integer. In contrast, the Cauchy problem for

$$
U^{(\alpha)}-A U=T
$$

is well set for any $\alpha>0$ but again none of the propagators is of exponential growth at $\infty$.

6. The case $0<\alpha \leqq 2$.

TheOREM 6.1. The Cauchy problem for the equation

$$
U^{(\alpha)}-A U=T
$$

is well set and the propagator $S$ belongs to the space

$$
\left(\mathscr{S}_{f}^{\prime}\left(\Gamma_{\omega}\right)\right)(\mathscr{C}(E, D(A))
$$

if and only if $R\left(\lambda^{\alpha} ; A\right)$ exists for $\operatorname{Re} \lambda>\omega$ and for each $a>\omega$ there exists a polynomial $p \geqq 0$ such that

$$
\left\{(1+p(|\lambda|))^{-1} R\left(\lambda^{\alpha} ; A\right) ; \operatorname{Re} \lambda>a\right\}
$$

is equicontinuous in $\mathscr{\ell}(E, D(A))$ (or in $\mathscr{L}(E))$.

Proof. The necessity of the conditions can be proved as in Theorem (5.2) by showing that $\mathbb{R} S=R\left(\lambda^{\alpha} ; A\right)$ and then using Lemma 5.1. As for the sufficiency, it follows from equicontinuity of (6.2), from the considerations opening $\S 5$ and again from Lemma 5.1 that

$$
R\left(\lambda^{\alpha} ; A\right)=\mathbb{2} S
$$

where $S$ is a distribution in $\left(\mathscr{S}_{f}^{\prime}\left(I_{\omega}\right)\right)(\mathscr{L}(E, D(A))$ with support in $t \geqq 0$. Let now $Z=S^{(\kappa)}-A S$. Since $\mathfrak{Q} Z=I$, we see that $Z=\delta \otimes I$, which shows that $S$ satisfies (2.3); then $S * T$ satisfies (6.1) for any $T \in \mathscr{D}^{\prime}(E)$. It only remains then the question of uniqueness of solutions of $(6.1)$, that is to verify (b) (or $\left.b^{\prime}\right)$ of $\S 2$. Let then $u(\cdot)$ be a $C^{\infty}, D(A)$-valued function, $u(t)=0$ for $t \leqq a$ and such that

$$
u^{(\alpha)}(t)-A u(t)=0
$$


for $t \leqq b, a \leqq b$ (we may plainly assume that $b=0$ ). Let now $\phi \in \mathscr{D}$, $\varphi(t)=1$ in $[a, b]$. Then

$$
(\varphi u)^{(\alpha)}(t)-A(\varphi u)(t)=g(t)
$$

where $g(t)$ is still zero for $t \leqq 0\left((\phi u)^{(\alpha)}(t)=u^{(\alpha)}(t)\right.$ for $\left.t \leqq 0\right)$ but it also vanishes for large $t$. We take Laplace transforms of both sides of (6.3) and obtain, after multiplying by $R\left(\lambda^{\alpha}, A\right)$

$$
(\mathscr{Q}(\varphi u))(\lambda)=R\left(\lambda^{\alpha} ; A\right)(\Omega g)(\lambda) .
$$

We use now the (easily verifiable) fast that the set $\{(\mathbb{R} g)(\lambda) ; \operatorname{Re} \lambda \geqq 0\}$ is bounded in $E$, the relation (6.4) and equicontinuity of the set (6.2) to deduce that if $\operatorname{Re} \lambda>a$ the set

$$
\left\{(1+p(|\lambda|))^{-1}(\mathcal{Q}(\varphi u))(\lambda) ; \operatorname{Re} \lambda \geqq a\right\}
$$

is bounded in $E$ ( $p$ the same polynomial in (6.2)). Applying Lemma 5.1 we see that $\varphi u$ (hence $u$ ) is zero for $t \leqq 0$. This ends the proof of Theorem 6.1 .

Theorem 6.1 reduces for $\alpha=1, E$ a Banach space to a result of Lions (see [7], Théorèmes 6.1, 5.1 and Corollaire 4.1) that gives necessary and sufficient conditions for the Cauchy problem for $U^{\prime}-A U=T$ to be well set in terms of the theory of distribution semi-groups of exponential increase at $\infty$.

The author is grateful to Professor J. L. Lions for bringing these problems to his attention and for most valuable suggestions, as well as to the referee for spotting several errors in the first version of the paper.

Results in this article have been announced (under the title "Sur quelques équations différentielles pour les distributions vectorielles) in C. Rendus Acad. Sci. Paris 268 (1969), 707-709.

\section{BIBLIOGRAPHY}

1. N. Bourbaki, Éléments de Mathematique, fasc. XV, Espaces Vectoriels Topologiques, ch. I, II, 2nd edition, Hermann, Paris, I966.

2. - Eléments de Mathematique, fasc. XVIII, Espaces Vectoriels Topologiques, ch. III-V, Hermann, Paris, 1955.

3. H. O. Fattorini, Ordinary differential equations in linear topological spaces, I, J. Diff. Equations 5 (1969), 72-105.

4. - Ordinary differential equations in linear topological spaces, II J. Diff. Equations 6 (1969), 50-70.

5. W. B. Ford, Studies on divergent series and summability (Univ. of Michigan Press, Ann Arbor, 1916) and The asymptotic development of functions defined by MacLaurin series (Univ. of Michigan Press, Ann Arbor, 1936), reprinted by Chelsea, New York, 1960. 
6. J. L. Lions, Supports dans la transformation de Laplace, J. d'Analyse Mathematique, II (1952/53), 369-380.

7. - Les semigroupes distributions, Portugalia Math. 19 (1960), 141-164.

8. L. Schwartz, Théorie des distributions a valeurs vectorielles, I, Ann. Inst. Fourier, VII (1957), 1-141.

9. — Théorie des distributions a valeurs vectorielles, II, Ann. Inst. Fourier, VIII (1958), 1-209.

10. - Théorie des Distributions, nouvelle edition, Hermann, Paris, 1966.

11. E. Hille and R. S. Phillips, Functional analysis and semi-groups, Amer. Math. Soc., Providence, 1957.

12. J. Chazarain, Problèmes de Cauchy au sens des distributions vectorielles et applications, C. R. Acad. Sci., Paris 266 (1968), 10-13.

Received July 1, 1968, and in revised form October 28, 1968, and April 18, 1969. The preparation of this paper was sponsored by the Office of Naval Research Under Contract NONR 233(76). Reproduction in whole or in part is permitted for any purpose of the United States Government.

University of CAlifornia, Los Angeles 


\section{PACIFIC JOURNAL OF MATHEMATICS}

\section{EDITORS}

\author{
H. SAMELSON \\ Stanford University \\ Stanford, California 94305 \\ Richard Pierce \\ University of Washington \\ Seattle, Washington 98105
}

J. DUGUNDJI

Department of Mathematics

University of Southern California

Los Angeles, California 90007

BASIL GORDON*

University of California

Los Angeles, California 90024

\section{ASSOCIATE EDITORS}
E. F. BECKENBACH
B. H. NeUmanN
F. WOLF
K. YoSHIDA

\section{SUPPORTING INSTITUTIONS}

UNIVERSITY OF BRITISH COLUMBIA CALIFORNIA INSTITUTE OF TECHNOLOGY

UNIVERSITY OF CALIFORNIA MONTANA STATE UNIVERSITY

UNIVERSITY OF NEVADA

NEW MEXICO STATE UNIVERSITY

OREGON STATE UNIVERSITY

UNIVERSITY OF OREGON

OSAKA UNIVERSITY

UNIVERSITY OF SOUTHERN CALIFORNIA
STANFORD UNIVERSITY

UNIVERSITY OF TOKYO

UNIVERSITY OF UTAH

WASHINGTON STATE UNIVERSITY

UNIVERSITY OF WASHINGTON

AMERICAN MATHEMATICAL SOCIETY CHEVRON RESEARCH CORPORATION TRW SYSTEMS

NAVAL WEAPONS CENTER 


\section{Pacific Journal of Mathematics}

\section{Vol. 32, No. $1 \quad$ January, 1970}

Robert Alexander Adams, Compact Sobolev imbeddings for unbounded domains ........................................ 1

Bernhard Amberg, Groups with maximum conditions .................. 9

Tom M. (Mike) Apostol, Möbius functions of order k............... 21

Stefan Bergman, On an initial value problem in the theory of two-dimensional transonic flow patterns ................... 29

Geoffrey David Downs Creede, Concerning semi-stratifiable spaces ...... 47

Edmond Dale Dixon, Matric polynomials which are higher

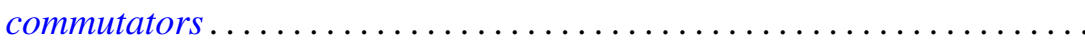

R. L. Duncan, Some continuity properties of the Schnirelmann density.

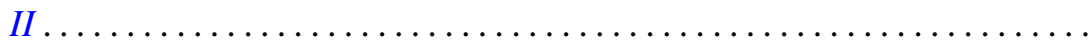

Peter Larkin Duren and Allen Lowell Shields, Coefficient multipliers of $H^{p}$

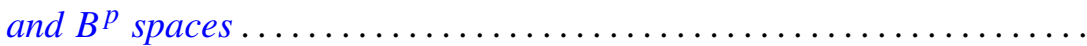

Hector O. Fattorini, On a class of differential equations for vector-valued

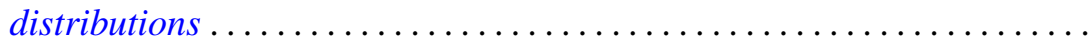

Charles Hallahan, Stability theorems for Lie algebras of derivations. . . . . . 105

Heinz Helfenstein, Local isometries of flat tori ................ 113

Gerald J. Janusz, Some remarks on Clifford's theorem and the Schur

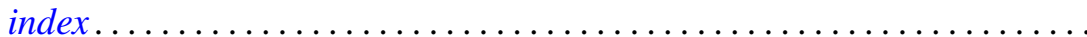

Joe W. Jenkins, Symmetry and nonsymmetry in the group algebras of discrete groups. ...

Herbert Frederick Kreimer, Jr., Outer Galois theory for separable

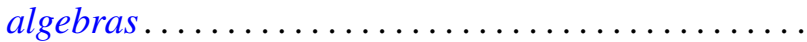

D. G. Larman and P. Mani, On visual hulls

R. Robert Laxton, On groups of linear recurrences. II. Elements of finite order.

Dong Hoon Lee, The adjoint group of Lie groups ...

James B. Lucke, Commutativity in locally compact rings

Charles Harris Scanlon, Rings of functions with certain Lipschitz

$$
\text { properties ............................... }
$$

Binyamin Schwarz, Totally positive differential systems .

James McLean Sloss, The bending of space curves into piecewise helical curves.

James D. Stafney, Analytic interpolation of certain multiplier spaces ...

Patrick Noble Stewart, Semi-simple radical classes.......

Hiroyuki Tachikawa, On left $\mathrm{QF}-3$ rings ...................... 255

Glenn Francis Webb, Product integral representation of time dependent nonlinear evolution equations in Banach spaces.... . . . 